\title{
Application of Stereo PIV on a Supersonic Parachute Model
}

\author{
Mark P. Wernet* \\ NASA Glenn Research Center, Cleveland, $\mathrm{OH}$ \\ Randy J. Locke ${ }^{\dagger}$ and Adam Wroblewski ${ }^{\ddagger}$ \\ ASRC, Inc., Cleveland, $\mathrm{OH}$ \\ Anita Sengupta ${ }^{\S}$ \\ Jet Propulsion Laboratory, California Institute of Technology, Pasadena, CA
}

The Mars Science Laboratory (MSL) is the next step in NASA's Mars Exploration Program, currently scheduled for 2011. The spacecraft's descent into the Martian atmosphere will be slowed from Mach 2 to subsonic speeds via a large parachute system with final landing under propulsive control. A Disk-Band-Gap (DBG) parachute will be used on MSL similar to the designs that have been used on previous missions, however; the DBG parachute used by MSL will be larger $(21.5 \mathrm{~m})$ than in any of the previous missions due to the weight of the payload and landing site requirements. The MSL parachute will also deploy at higher Mach number (M 2) than previous parachutes, which can lead to instabilities in canopy performance. Both the increased size of the DBG above previous demonstrated configurations and deployment at higher Mach numbers add uncertainty to the deployment, structural integrity and performance of the parachute. In order to verify the performance of the DBG on MSL, experimental testing, including acquisition of Stereo Particle Imaging Velocimetry (PIV) measurements were required for validating CFD predictions of the parachute performance. A rigid model of the DBG parachute was tested in the 10x10 foot wind tunnel at GRC. Prior to the MSL tests, a PIV system had never been used in the 10x10 wind tunnel. In this paper we discuss some of the technical challenges overcome in implementing a Stereo PIV system with a $750 \times 400 \mathrm{~mm}$ field-of-view in the 10x10 wind tunnel facility and results from the MSL hardshell canopy tests.

\section{Introduction}

$\mathrm{T}^{\mathrm{H}}$ HE Mars Science Laboratory (MSL) is the next step in NASA's long-term effort of robotic exploration of the Martian surface. The Mars Science Laboratory, the largest rover ever delivered to the planet, will determine if Mars was ever a suitable environment for supporting microbial life. MSL will use a new Entry, Descent and Landing (EDL) system, designed to deliver the payload to previously unobtainable landing sites and with higher precision than previous landers. The spacecraft's descent into the Martian atmosphere will be slowed from Mach 2 to subsonic speeds via a large parachute system with final landing under propulsive control. A Disk-Band-Gap (DBG) parachute will be used on MSL similar to the designs that have been used on Viking, Mars Exploration Rover and Pathfinder missions. The DBG parachute used by MSL will be larger $(21.5 \mathrm{~m})$ than in any of the previous missions due to the weight of the payload and required precision to surface target. ${ }^{1,2}$ Increasing the size of the DBG above previous demonstrated configurations adds some uncertainty to the deployment, structural integrity and performance of the parachute. More specific details of the parachute, including construction materials and design guidelines can be found in reference 1. Tests of the Viking parachute prior to the mission showed a post-inflation parachute behavior termed "area oscillation" characterized by small, periodic in-folds in the band region (the leading edge ring) resulting in projected area fluctuations and changes in drag performance. ${ }^{3,4}$ The "area oscillations" are more pronounced above Mach 1.5, where MSL will spend 5-10 seconds during deceleration. The Viking tests determined that this phenomenon did not adversely affect the parachute in the Viking qualification Mach number range. Although the Viking program identified possible causes of area oscillation, a fundamental physical understanding of the forebody wake/parachute interaction was not developed. In order to verify the performance of the DBG on MSL, additional testing is required for validating the CFD predictions.

\footnotetext{
* Senior Research Engineer, Optical Instrumentation Technology and NDE Branch, Associate Fellow AIAA

† Senior Research Engineer, Optical Instrumentation Technology and NDE Branch

${ }^{\ddagger}$ Research Engineer, Optical Instrumentation Technology and NDE Branch

$\S$ Senior Systems Engineer, Entry Descent, Landing and Advanced Technologies, Senior Member AIAA
} 
A three-phase test program has been implemented to better understand the stability and performance of the band gap parachutes under the expected flight envelop of MSL: 1) Rigid Canopy tests; 2) Flexible Canopy tests; and 3) Fluid Structure Interaction Analysis Validation. ${ }^{5}$ Parts 1 and 2 involve wind tunnel testing of scaled parachute models. The topic of this paper covers portion 1 of the testing on a rigid DBG parachute model in the Glenn Research Center's (GRC) 10x10 foot supersonic wind tunnel. In part 2 of the test program, high speed Schlieren, Photogrammetry and Stereo PIV measurements on a flexible canopy model are required in order to perform the Fluid-Structure Interaction analysis in the final phase of the program.

The instability range of interest for the MSL parachute occurs in the Mach 1.5 to 2.5 range, which is mostly within the normal operating range of the 10x10 (Mach 2-3.5). Prior to the MSL tests, a PIV system had never been used in the 10x10 wind tunnel. Several challenges needed to be overcome before installation of the PIV system: Optical Access, flow seeding and laser sheet delivery into the test section. One additional technical challenge was the MSL program request for a Stereo PIV system field-of-view of $750 \times 400 \mathrm{~mm}$, which is much larger than any other previous field-of-view used at GRC.

\section{10x10 Wind Tunnel}

The test section of the $10 \times 10$ foot wind tunnel is $3.0 \mathrm{~m}$ high by $3.0 \mathrm{~m}$ wide by $12.2 \mathrm{~m}$ long and can accommodate large-scale models, full-scale engines and aircraft components. The 10x10 was specifically designed to test supersonic propulsion components such as inlets and nozzles, propulsion system integration, and full-scale jet and rocket engines. It can operate as a closed-loop system (aerodynamic cycle) or open-loop system (propulsion cycle), reaching test section speeds of Mach 2.0 to 3.5 and very low speeds from 0 to Mach 0.36 . There is also continuous operation across the entire speed and altitude regime, offering users greater flexibility and productivity during testing.

In the propulsion cycle, the tunnel operates by continuously drawing outside air through a very large air dryer to remove the moisture and exhausting it back to the outside environment. During the aerodynamic cycle, the tunnel runs as a variable density facility that can simulate pressure altitude conditions ranging from 50,000 to 154,000 ft. Dry air is added as needed to maintain test conditions.

The 10x10 has two independent Schlieren systems, one upstream and one downstream, each with $83.8 \mathrm{~cm}$ diameter window ports mounted in a pair of tunnel wall turntables. The turntables permit adjusting the Schlieren system viewing location and height over a limited range within tunnel test section. The downstream Schlieren system was used for the MSL test. A high speed video (4 kHz, 800x600 pixels) camera was used to record the Schlieren images of the rigid canopy bow shock oscillations. The field-of-view of the Schlieren system covered a larger area $(1328 \times 966 \mathrm{~mm})$ than that of the Stereo PIV system.

\section{Model Hardware}

The MSL hardshell model, consisting of a rigid canopy and a capsule, was fabricated by NASA Ames and initially tested in their $9 \mathrm{x} 7 \mathrm{foot}$ wind tunnel. ${ }^{2}$ The rigid canopy was manufactured from aluminum and is $30 \mathrm{~cm}$ in diameter and $5 \mathrm{~mm}$ thick. The canopy was mounted to a 6 -axis force balance, of which only the axial force was recorded for the MSL tests. There were 12 straps supporting the band at the front of the parachute (in the flexible canopy there are actually 24 suspension lines) and a vent port at the apex of the canopy. The rigid canopy was mounted on the floor strut which permitted both angle of attack and height adjustments.

The capsule models were mounted on a fixed ceiling strut to avoid blocking the PIV light sheet entering from a port in the tunnel floor, see figure 1 . Three capsule models with different diameters (D) and angles of attack $(\alpha)$ were used: 1) $104 \mathrm{~mm}$ diameter, $\alpha=0^{\circ}$; 2) $82.5 \mathrm{~mm}$ diameter, $\alpha=0^{\circ}$; and 3) $82.5 \mathrm{~mm}$ diameter, $\alpha=10^{\circ}$. The spacing between the capsule and the canopy leading edge was adjusted to keep $\mathrm{x} / \mathrm{D}$ fixed at 10 . In addition, capsule only cases were also run to characterize the turbulent wake flow behind the capsule. The full suite of test cases that were run is shown in Tables I and II, where $\mathrm{D}_{\mathrm{p}}$ is the nominal diameter of the full scale parachute being simulated in the test. Subsequent to the initiation of testing of the MSL hardware, the MSL program selected an actual flight parachute size of $21.5 \mathrm{~m}$. The test models used here bracket the selected flight parachute size. 


\begin{tabular}{|c|c|c|c|c|c|c|}
\hline & & & & \multicolumn{3}{|c|}{ Mach Number } \\
\hline $\mathbf{x} / \mathbf{D}$ & $\mathbf{D}_{\mathbf{p}}$ & $\mathbf{D}$ & Capsule $\boldsymbol{\alpha}$ & $\mathbf{2 . 0}$ & $\mathbf{2 . 2}$ & $\mathbf{2 . 5}$ \\
\hline 10 & $19 \mathrm{~m}$ & $104 \mathrm{~mm}$ & $0^{\circ}$ & $\checkmark$ & $\checkmark$ & $\checkmark$ \\
\hline 10 & $25 \mathrm{~m}$ & $82.5 \mathrm{~mm}$ & $0^{\circ}$ & $\checkmark$ & $\checkmark$ & $\checkmark$ \\
\hline 10 & $25 \mathrm{~m}$ & $82.5 \mathrm{~mm}$ & $10^{\circ}$ & $\checkmark$ & $\checkmark$ & $\checkmark$ \\
\hline
\end{tabular}

Table I: Capsule-Canopy test matrix.

\begin{tabular}{|c|c|c|c|c|c|c|}
\hline & & & & \multicolumn{3}{|c|}{ Mach Number } \\
\hline $\mathbf{X} / \mathbf{D}$ & $\mathbf{D}_{\mathbf{p}}$ & $\mathbf{D}$ & Capsule $\boldsymbol{\alpha}$ & $\mathbf{2 . 0}$ & $\mathbf{2 . 2}$ & $\mathbf{2 . 5}$ \\
\hline 10 & $19 \mathrm{~m}$ & $104 \mathrm{~mm}$ & $0^{\circ}$ & $\checkmark$ & $\checkmark$ & $\checkmark$ \\
\hline 10 & $25 \mathrm{~m}$ & $82.5 \mathrm{~mm}$ & $10^{\circ}$ & $\checkmark$ & $\checkmark$ & $\checkmark$ \\
\hline
\end{tabular}

Table II: Capsule-Only test matrix.

\section{PIV System Installation}

Floor and ceiling plates equipped with various sized optical access ports already exist for the 10x10. A floor plate with a $75 \mathrm{~mm}$ diameter window port on the tunnel centerline was selected for introducing the laser light sheet into the test section. The large Schlieren windows would have provided easy optical access for the PIV cameras, however; the MSL test requirements for both high speed Schlieren and PIV measurements during the tests dictated that new PIV viewing window ports de installed. The new PIV window ports, $30 \mathrm{~cm}$ in diameter, were designed, fabricated and installed in the tunnel sidewall turntables (opposite the location of the Schlieren window ports) in order to provide the required PIV system optical access.

The PIV cameras were mounted on each side of the wind tunnel and viewed the illuminated light sheet plane through the new PIV windows, see figure 1. The laser light sheet (dual head $400 \mathrm{~mJ} / \mathrm{pulse}$ Nd:YAG operating at $10 \mathrm{~Hz}$ and $532 \mathrm{~nm}$ ) entered the test section through a $75 \mathrm{~mm}$ diameter port in the tunnel floor and impinged on the tunnel ceiling. The light sheet (approximately $2 \mathrm{~mm}$ thick by $65 \mathrm{~cm}$ wide at the tunnel centerline) was centered on the tunnel floor and ceiling, with the plane of the light sheet aligned with the stream-wise axis of the tunnel, see figure 2. High resolution (4008x2672 pixel) frame-straddling cameras, equipped with 105 mm focal length lenses, were used to provide the required large field of view, and yet provide $<3 \mathrm{~mm}$ spatial resolution in the processed velocity vector maps. The cameras were oriented with the $4 \mathrm{k}$ pixel axis along the vertical and equipped with $45 \mathrm{~mm}$ diameter Uniblitz electromechanical shutters to shorten the duration of the $2^{\text {nd }}$ exposure in the PIV image frame pairs. The resulting Stereo PIV system had a 750x390mm (HxW) field-of-view centered vertically within the wind tunnel test section. The orientation of the PIV cameras relative to the light sheet propagation direction yielded a nearly forward scattering configuration $\left( \pm 62^{\circ}\right)$, in order to maximize the sensitivity of the PIV cameras to the submicron seed particles in the flow. The PIV system was controlled via an in-house developed PIVACQ program, which provided data acquisition rates of image frame pairs at $2 \mathrm{~Hz}$. PIVACQ streams the data from the two PIV cameras via a CameraLink interface directly to two internal SATA RAID 0 hard drives for real time data acquisition (at $88 \mathrm{Mbytes} / \mathrm{sec}$ ) and unlimited sequence length (limited only by available hard drive space). PIVACQ can also IRIG-B timestamp the acquired image files for later synchronization with other data acquisition systems. PIVACQ operates the lasers at $10 \mathrm{~Hz}$ and drives the Uniblitz shutters on each PIV camera at $2 \mathrm{~Hz}$ to block the light of the intervening laser pulses from exposing the camera sensors during image readout. The proposed test plan included acquiring 2400 PIV image frame pairs in order to build accurate ensemble averages of the canopy bow shock oscillations.

\section{Flow Seeding}

After reviewing all of the available options, it was determined that the best method for introducing the PIV seed material into the 10x10 test section was through the existing tunnel heating system. The tunnel heating system consists of three concentric rings of $9.5 \mathrm{~mm}$ diameter burner nozzles fed by natural gas. Only the core ring of heaters was used for introducing the seeding. The natural gas supply line was replaced with a $150 \mathrm{~mm}$ diameter line connected to a high pressure vessel mounted below the tunnel settling chamber, see figure 3 .

Due to the high velocity flow anticipated in the test section, submicron sized seed particles were required in order to accurately follow the flow. The flow was seeded using two Vicount 5500 smoke generators, which generate condensed mineral oil droplets in the range of $0.2-0.3 \mu \mathrm{m}$ in diameter. The smoke generators were mounted inside a 
large pressure vessel located below the tunnel settling chamber which was equipped with shop air lines to help drive the seed material from the vessel up through the tunnel heater ducting and into the tunnel. The tunnel was operating below atmospheric pressure for these MSL runs, which simplified the process of injecting the seed material into the tunnel. The Vicount units provided high quality, uniform flow seeding, yielding well resolved particle images, as shown in figure 4. Although the seed concentration appears to be low in the capsule wake region (compared to the wake shock and canopy shock regions), there is actually plenty of seed material to provide good PIV correlation results.

\section{Stereo PIV Calibration \& Processing}

A vellum calibration target measuring $610 \times 762 \mathrm{~mm}$ with $2 \mathrm{~mm}$ dots on $10 \mathrm{~mm}$ centers was used to calibrate the Stereo PIV system. A 9-plane calibration was performed covering the range of $\pm 2 \mathrm{~mm}$ in $0.5 \mathrm{~mm}$ increments. ${ }^{6}$ A Calibration Verification operation was performed to ensure that the central calibration plane coincided with the plane of the light sheet. ${ }^{7}$ No improvement in the data was obtained by using the calibration verification (the plane of the calibration target was well aligned with the light sheet plane).

The PIV image data were processed using Symmetric Phase Only Filtering (SPOF) and multi-pass processing: a first pass with $64 x 64$ pixel subregions on 32 pixel centers followed by a $2^{\text {nd }}$ pass with $64 x 64$ pixel subregions on 16 pixel centers. $^{8}$ Two additional passes with Subregion Distortion processing yielded the final velocity vector maps. The $75 \%$ overlap is larger than desired but yielded higher quality vector maps for comparison with CFD predictions. The SPOF processing yielded high quality vector maps in cases where the PIV image data were corrupted by flare light from the canopy or refraction effects of strong shocks not related to the flow of interest. The processed velocity vector maps had a spatial resolution of $2.5 \mathrm{~mm}$. The large format of the ES-11000 cameras used here (4008x2672 pixels) resulted in some minor vignetting in the periphery of the images. The vignetting was exacerbated by the two leaf mechanical shutter used to control the camera exposures. The processed PIV data sets were then mapped into the tunnel coordinate system as shown in figure 5 , where the origin is located at the major diameter of the capsule.

\section{CFD Prediction}

CFD predictions of the MSL hardshell model were being performed under a parallel effort to use CFD to predict/understand the cause of the area oscillations observed in DBG parachutes. A CFD prediction of the capsulecanopy configuration was computed prior to the PIV testing. One of the main objectives of the current test was to provide benchmark data for validating the computer model. A Detached Eddy Simulation (DES) was used to predict the capsule wake interaction with the rigid disk band-gap parachute at a Reynolds Number per meter of $\mathrm{Re} / \mathrm{m}=9.9 \times 10^{6}$ and Mach $2 .{ }^{9}$ The main result from the simulation was that the canopy over-pressurizes and expels the excess gas, causing the bow shock to move axially fore and aft in the flow. The pressurization is strongly correlated with the bow shock motion and the axial drag on the parachute. More details of the simulation and results can be found in reference 9. A sample picture from a single time-step of the DES result is shown in figure 6, where the PIV system field-of-view is shown overlaid on the simulation domain. The CFD prediction shown here does not include the actual tunnel hardware ceiling strut or floor strut, but otherwise matches the experimental hardware used and flow field conditions in the wind tunnel tests. The PIV system field-of-view was selected to capture the canopy bow shock, but also contains the shocks generated from the capsule wake at the bottom and top of the field-of-view.

\section{Results \& Discussion}

The Vicount smoke generators provided uniform high quality seeding in the central region of the tunnel test section. The windows of the tunnel remained clean throughout the entire run, becoming only slightly coated with oil after several hours of runtime. Condensation in the tunnel resulted in a gray background haze in the PIV images. Using the tunnel dryers, the tunnel condensation was reduced to nearly zero at a dewpoint of $12^{\circ} \mathrm{C}$. Operating at this dewpoint yielded the highest quality PIV images.

The complete set of models and conditions shown in Tables I and II was completed. Only data from the Mach 2.0 case will be presented here. Data were collected at several different $\mathrm{Re} / \mathrm{m}$ during the test program. The PIV data presented here were collected at $\mathrm{Re} / \mathrm{m}=9.8 \times 10^{6}$. The Capsule-Only case results for the Mach $2.0, \mathrm{D}_{\mathrm{p}}=19 \mathrm{~m}$ case are shown in figure 7 where the processed PIV data have been placed in the tunnel coordinate system. All 2400 of the velocity vector maps were used to compute these ensemble average results. The canopy was lowered to $35.5 \mathrm{~cm}$ off of the tunnel floor for the capsule only configurations. The plot of the turbulence intensity in figure 7a shows very high quality flow ( $1 \%$ turbulence) behind the capsule wake shock. This agrees with results obtained in an early 
checkout run of the Stereo PIV system in the 10x10 where an empty test section flow was measured. In those empty test section measurements, the freestream turbulence levels in the 10x10 test section were less than $1 \%$ and the flow angularity was less than $0.25^{\circ}$. The streamwise velocity component in Figure $7 \mathrm{~b}$ shows some remnants of the wake from the ceiling strut. The bottom half of the flow field is very clean. The vertical velocity component in figure 7c shows some turning in front of the capsule wake shocks. The cross-stream velocity component in figure $7 \mathrm{~d}$ (out of the plane of the light sheet) shows very small fluctuations in velocity only over the range of $\pm 10 \mathrm{~m} / \mathrm{s}$. Here the wakes from the roughness of the ceiling strut are more readily observed.

Results from the Capsule-Canopy, Mach 2.0, $\mathrm{D}_{\mathrm{p}}=19 \mathrm{~m}$ configuration are shown in figure 8, where again the processed PIV data are placed in the tunnel coordinate system. All 2400 of the velocity vector maps were used to compute these ensemble average results. The axial velocity component plot in figure 8a clearly shows the canopy bow shock and the capsule wake. The range of oscillation of the canopy bow shock cannot be discerned from this plot. The vertical and out-of-plane velocity component plots are very similar to the capsule only cases with the exception of the canopy bow shock.

\section{A. Phase Averaging of the Canopy Bow Shock}

In the planning stages of the experiment, it was proposed that axial force balance measurements would be used to correlate the PIV measurements with the instantaneous load on the canopy. The CFD prediction of the capsulecanopy flow showed a strong correlation between the load on the canopy and the axial location of the shock. The further upstream the shock, the more over-pressurization in the canopy and hence, the higher the axial force on the canopy. IRIG-B timestamps were used to synchronize the $2 \mathrm{~Hz}$ PIV measurements with the $10 \mathrm{kHz}$ axial force balance digitization records. High speed Schlieren image data (4 kHz framing rate) with IRIG-B time-stamps were also collected during the experiment and these images were processed to determine the canopy shock front location as a function of time. Comparison of the shock front location records with axial force balance readings revealed that there was not always a 1:1 linkage between the shock location and the force reading on the canopy, see figure 9. It was also determined after the test that the axial force balance readings could be prone to ringing due to the configuration and length of the electrical connections to the transducer. Hence, the observed sinusoidal oscillations in axial force are probably erroneous. The Schlieren data records illustrate the true shock behavior.

Since the axial force measurements could not be used to reliably rectify the PIV measurements of the flow field, a phase averaging approach was used to generate an animation of the shock locations as a function of time. The processed PIV velocity vector maps clearly show the canopy shock front. The PIV vector maps were processed to determine the shock shape and axial location, see figure 10. A PDF of the shock locations across the 2400 measurement data record reveals that the shock has a preferred "mean" axial location at $87.0 \mathrm{~cm}$ downstream from the major axis of the capsule, see figure 11a. The shock makes excursions upstream and downstream of this mean location, as shown in figure 11b, where the number of measurements in the ensemble average shows a slight decrease at the min and max shock excursions. The axial locations of the shocks were then used to sort the vector maps into phase bins. The axial displacement range of the canopy shock was divided into 36 bins for the sorting process. Ensemble averages of all of the velocity maps in each phase bin were computed to generate the final animation. While this procedure enabled us to recreate the axial motion of the canopy shock, it also resulted in a $180^{\circ}$ phase ambiguity - we do not know which direction the shock is moving, only its position. Hence, in order to create a cyclical shock oscillation animation, a copy of the displacement profile was reversed and added to the end of the motion sequence to simulate the sinusoidal oscillations of the canopy shock. While the animation shows the full excursion of the canopy shock, the Schlieren data record shows that the shock does not always move in a sinusoidal pattern. The purpose of the animation was to identify the axial extent of the shock oscillations and to examine any significant changes in the flow field properties during the shock motion.

\section{B. Comparison with CFD}

The PIV data and DES simulation were at essentially the same conditions, except that the DES simulation did not contain the ceiling strut holding the capsule nor the floor strut holding the canopy. There are several features of the DES simulation that are readily compared with the PIV measurement results. In the DES, we are looking at essentially instantaneous time-steps in the capsule wake - canopy shock interaction. For the PIV results, we have used the phase binning approach to obtain ensemble averaged flow field estimates across the range of shock oscillations. However, we also have instantaneous PIV velocity vector maps for comparison with the DES results. Firstly, the capsule wake generates a shock wave as seen in figure 6. Figure 12 shows a plot of Mach number for both the DES and an instantaneous PIV result. In the DES result, the capsule wake oblique shocks are symmetric at 
$\pm 62^{\circ}$. In the PIV results, which are more clearly shown in the ensemble average plots in figure 8 , the top oblique shock is at $62^{\circ}$ while the lower shock is at $63.5^{\circ}$. The DES simulation does not include the ceiling strut used to hold the capsule in the wind tunnel tests. The wake of the strut is the most likely cause of the asymmetry in the PIV measured shock angles. The lower oblique shock should not be affected by the ceiling strut, yet this is the shock that agrees less with the DES result than the top oblique shock. Secondly, the general shape of the canopy bow shock appears different between the two. In the DES simulation the canopy shock is always highly distorted at the point where the capsule wake intersects the shock, and it appears that the higher velocity flow downstream of the shock is actually tunneling upstream ahead of the shock. However, in the PIV results, the canopy shock shape remains relatively flat, even at the intersection of the capsule wake and the canopy shock. Thirdly, we also observe in the instantaneous PIV result that the capsule wake flow appears to contain more small scale turbulence than the DES prediction. Finally, the range of shock motion is very different between the two cases. In the DES result - the canopy shock moves over a range of roughly $110 \mathrm{~mm}$. In the PIV result, the min-max excursions of the canopy shock are observed to cover the range of about $60 \mathrm{~mm}$, only half of the DES result. The DES results shown here do not include the ceiling and floor struts, which may impact some of the predicted flow physics.

\section{Moving Capsule-Canopy Configuration Transition}

The DES simulation results predict that the variations in the loading of supersonic disk-band-gap parachutes are caused by the interaction of the unsteady capsule wake with the canopy flow field. The time-varying momentum deficit in the wake causes the canopy to become over-pressurized, then rapidly eject the built-up excessive mass, and then refill. This cyclical process produces large variations in the axial force on the parachute. For the canopy only configuration, the canopy bow shock has a very stable location in front of the canopy. The addition of the capsule wake creates a momentum deficit in the incoming fluid which alters the shape of the bow shock, effectively increasing its standoff distance.

During the capsule only runs, the floor strut holding the canopy was lowered to $35.5 \mathrm{~cm}$ off of the tunnel floor, as shown in figure 7. The canopy was lowered or raised into position while the tunnel was running depending on the required configuration. During one of the configuration changes from capsule only to capsule-canopy, the PIV system acquired a sequence of image frame pairs. The processed PIV velocity vector maps show that as the canopy rises up into the PIV system field of view, the canopy bow shock is very steady at a mean location of $92.1 \mathrm{~cm}$ downstream from the capsule. As the canopy is raised further and intersects the capsule wake, the shock is energized and the instability is readily observed. Comparing this location with that obtained from figure 11a shows that the shock mean location shifts upstream by $5 \mathrm{~cm}$ when it is in the capsule wake. The PIV measurements confirm the DES prediction of a larger standoff distance for the canopy shock when in the capsule wake.

\section{Conclusions}

The near forward scattering configuration of the Stereo PIV system yielded good signal levels from the small seed particles. Calibration verification was used to ensure that the calibration was performed in the plane of the laser light sheet. The resulting PIV measurement error was nominally 1\%. PIV measurements in an empty test section checkout run verified the high quality flow in the $10 \times 10$ test section to be $<1 \%$ turbulence and $<0.25^{\circ}$ flow angularity. The PIV data processing strategy was optimized (employing SPOF and Subregion Distortion Processing) to minimize the effects of shocks and flare light in the recorded images. The processed PIV vector maps were used to compute both the ensemble averaged mean velocity profiles and also to generate phase averaged velocity maps of the shock locations during the canopy shock oscillations. The PIV data characterized the capsuleonly case ensemble averaged flow properties. For the Capsule-Canopy case, the measurements also characterized the range of motion of the canopy shocks, the capsule wake trajectory, and the turbulence scales in the wake flow. The PIV measurements were compared to a DES prediction of the capsule-canopy flow. In general, the PIV results agree with the DES prediction, differing mainly in the magnitude of the canopy-shock oscillation and oblique angles of the capsule wake shock. The DES prediction indicates a stronger capsule wake-canopy shock interaction than observed in the PIV results. The fidelity of the DES prediction is still evolving. More refinement of the turbulence models and inclusion of more details of the actual wind tunnel model hardware may affect the results.

\section{Acknowledgements}

Special thanks to Prof. Graham Candler for permission to use the DES results for the MSL hardware. The CFD predictions proved invaluable in determining the optimal PIV experimental configuration. We would also like to thank Al Linne and Jim Roeder for organizing, planning and implementing the MSL test program at GRC. We also recognize the efforts of Garret Clayo and Peter Eichele for the setup and operation of the PIV system. 


\section{References}

${ }^{1}$ Sengupta, A., Steltzner, A., Witkowski, A., Rowan, J., "An Overview of the Mars Science Laboratory Parachute Decelerator System,” Proceedings of the IEEE 2007 Aero Conf, March 2007, pp. 1-8.

${ }^{2}$ Sengupta, A., Steltzner, A., Comeaux, K., Candler, G., Pantano, C., Bell, J., "Supersonic Delta Qualification by Analysis Program for the Mars Science Laboratory Parachute Decelerator System”, AIAA 2007-2542, 19th AIAA Aerodynamic Decelerator Systems Technology Conference, May 2007.

${ }^{3}$ Reichenau, D.E.A., "Aerodynamic Characteristics of Disk-Gap-Band Parachutes in the Wake of Viking Entry Forebodies at Mach Numbers from 0.2 to 2.6,” AEDC-TR-72-78, AEDC, Arnold AFB, Tennessee, 1972.

${ }^{4}$ Steltzner, A., Cruz, J., Brunof, R. and Mitcheltree, R., “Opportunities and Limitations in Low Earth Subsonic Testing for Qualification of Extraterrestrial Supersonic Parachute Designs”, AIAA-2003-2135, $17^{\text {th }}$ AIAA Aerodynamic Decelerator Systems Technology Conference and Seminar, 19-22 May 2003.

${ }^{5}$ Lingard, J. S. and Darley, M. G., "Simulation of Parachute Fluid Structure Interaction in Supersonic Flow”, AIAA2005-1607, $18^{\text {th }}$ AIAA Aerodynamic Decelerator Systems Technology Conference and Seminar, 2003.

${ }^{6}$ Soloff, S.M., Adrian, R.J., and Liu, Z-C, "Distortion Compensation for Generalized Stereoscopic Particle Image Velocimetry,” Measurement Science and Technology, Vol. 8, No. 12, 1997, pp. 1441-1454.

${ }^{7}$ Bjorkquist, D.C., "Design and Calibration of a Stereoscopic PIV System,” Proc. 9th International Symposium on Applications of Laser Techniques to Fluid Mechanics, Vol. 1, Lisbon, Portugal, 1998, pp. 6.5.1-6.5.8.

${ }^{8}$ Wernet, M. P., "Symmetric Phase Only Filtering: a New Paradigm for DPIV Data Processing”, Meas. Sci. Technology, Vol. 16, 2005, pp. 601-618.

${ }^{9}$ Barnhardt, M., Drayna, T., Nompelis, I., Candler, G. V., Garrard, W., "Detached Eddy Simulations of the MSL Parachute at Supersonic Conditions”, AIAA-2007-2529, 19th AIAA Aerodynamic Decelerator Systems Technology Conference, 2007.

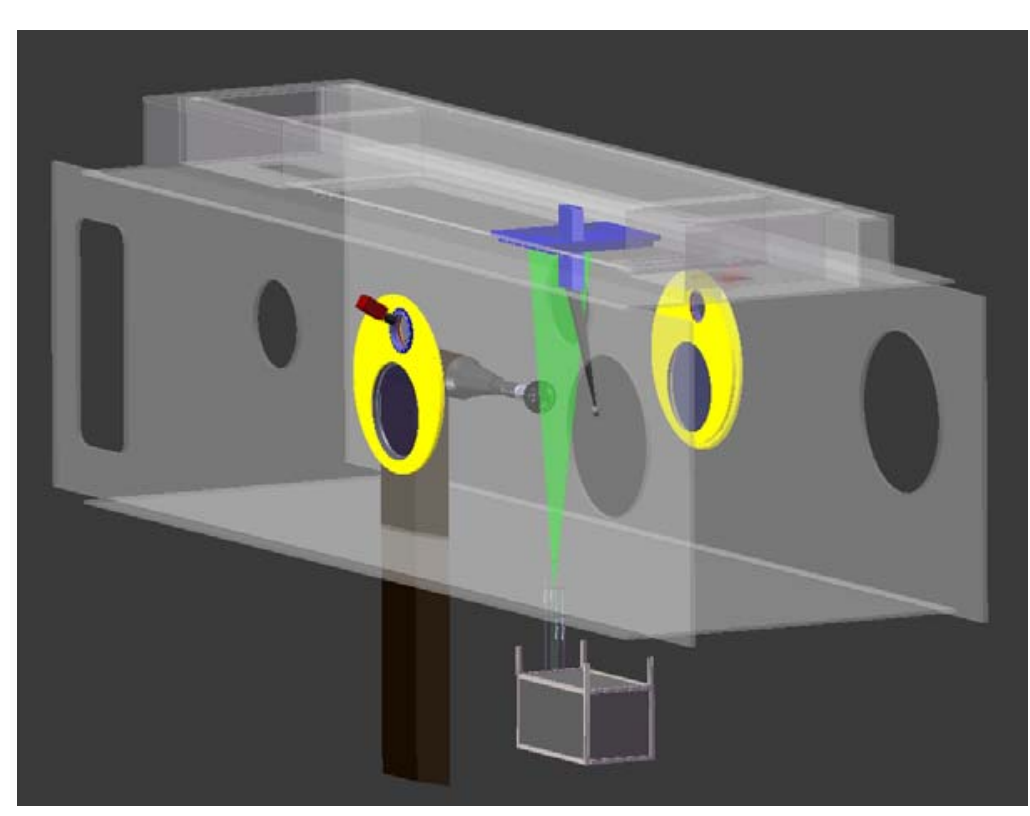

Figure 1: PIV system installation in the 10x10 Wind Tunnel, viewed from upstream. The PIV laser is contained in the housing suspended below the tunnel floor. New PIV window access ports were added into the sidewall turntables which hold the Schlieren windows.

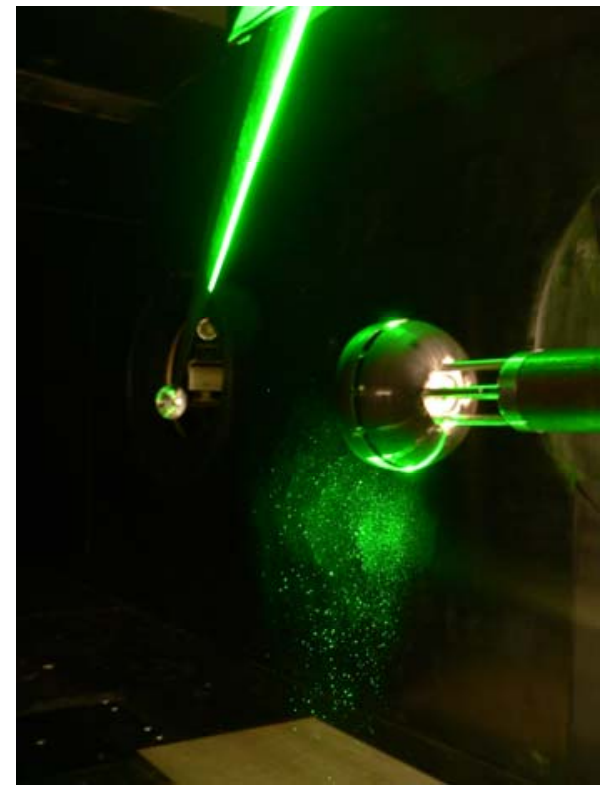

Figure 2: PIV system light sheet entering through a window port in the tunnel floor. View is from downstream of the canopy looking upstream at the ceiling strut supported capsule. 


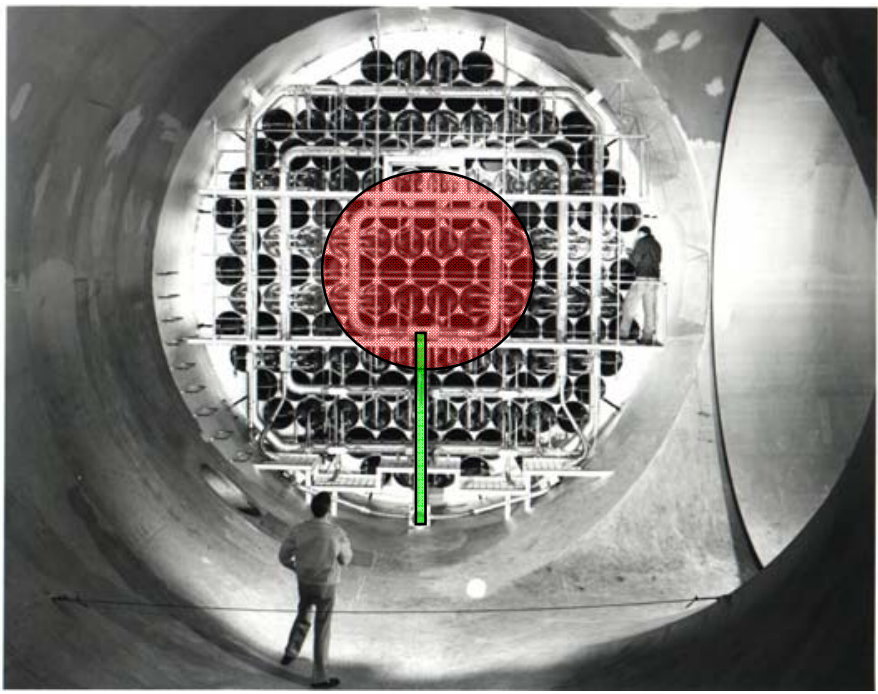

Figure 3: PIV seed material introduced into the tunnel through the tunnel heating system. Green pipe shows the feed line from the pressure vessel outside of the tunnel into the central ring of heaters, which are depicted by the red circle. Only the core ring of heaters was used for introducing the flow seeding.
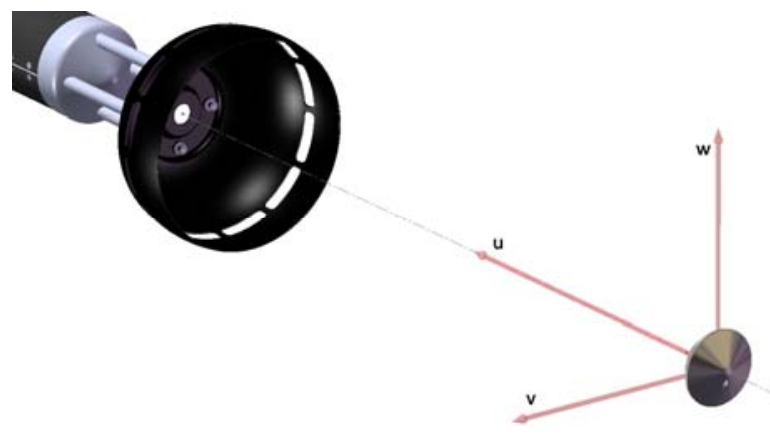

Figure 5: The MSL model coordinate system in the tunnel. The reference coordinate system shows the vertical velocity component $(\mathrm{w})$, the stream-wise velocity component (u) and the cross stream velocity component (v), which is out of the plane of the light sheet.

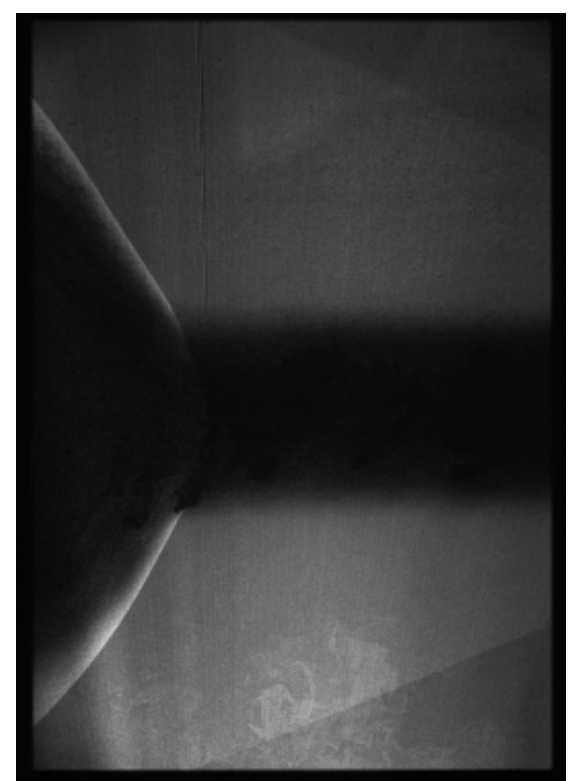

Figure 4: Raw PIV image illustrating flow seeding at the measurement plane in the tunnel. Canopy bow shock and capsule wake shock are readily observed.

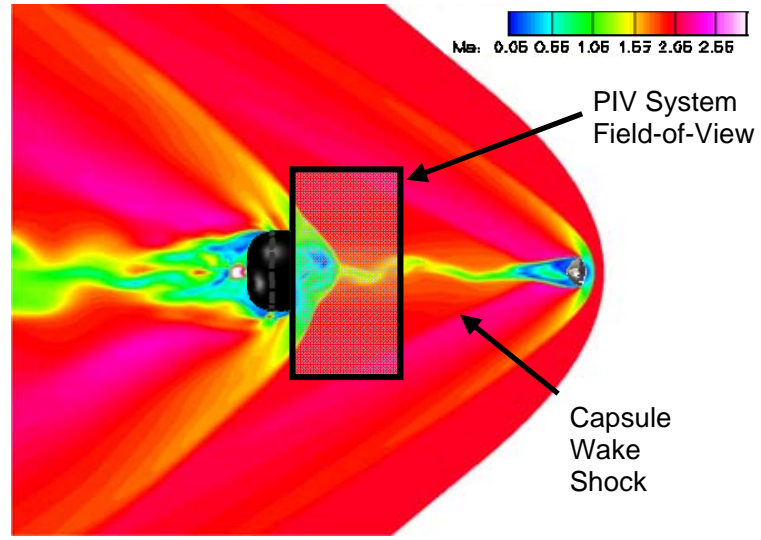

Figure 6: DES prediction of the capsule-canopy flow field colored by Mach number. The PIV system field of view is outlined in the figure. 
a)

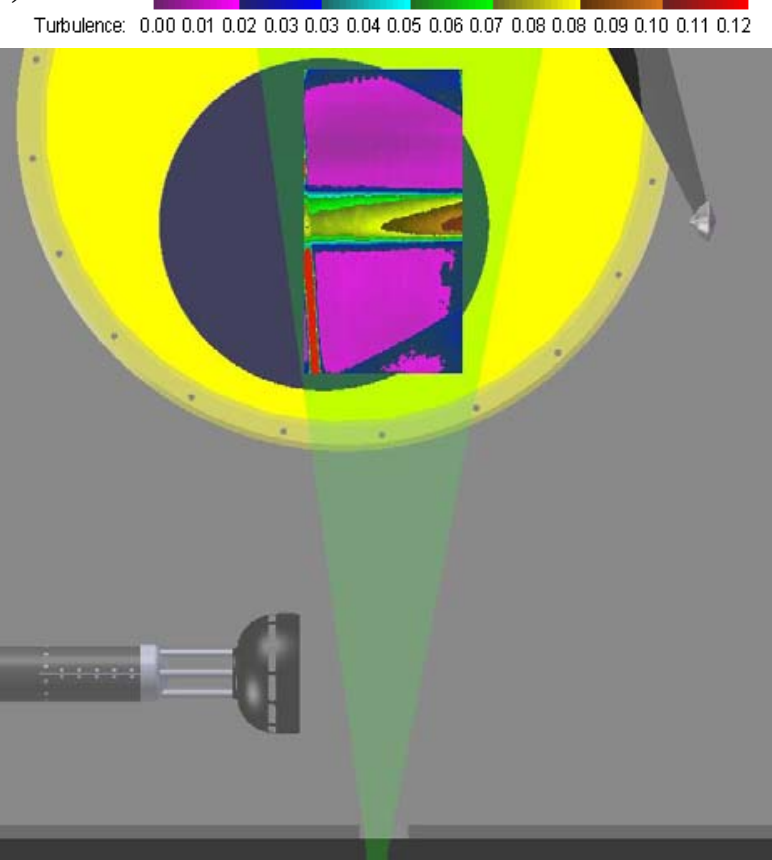

c) $\mathrm{vm} / \mathrm{s}-50-45-40-35-30-25-20-15-10-5 \quad 0 \quad 5 \quad 10152025 \quad 3035 \quad 4045 \quad 50$

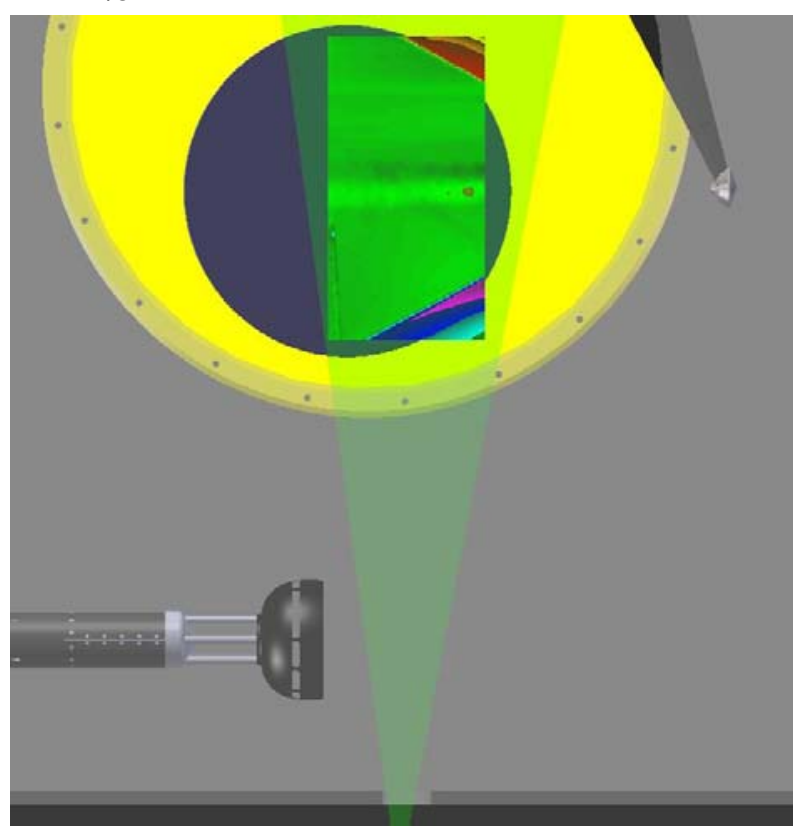

b)

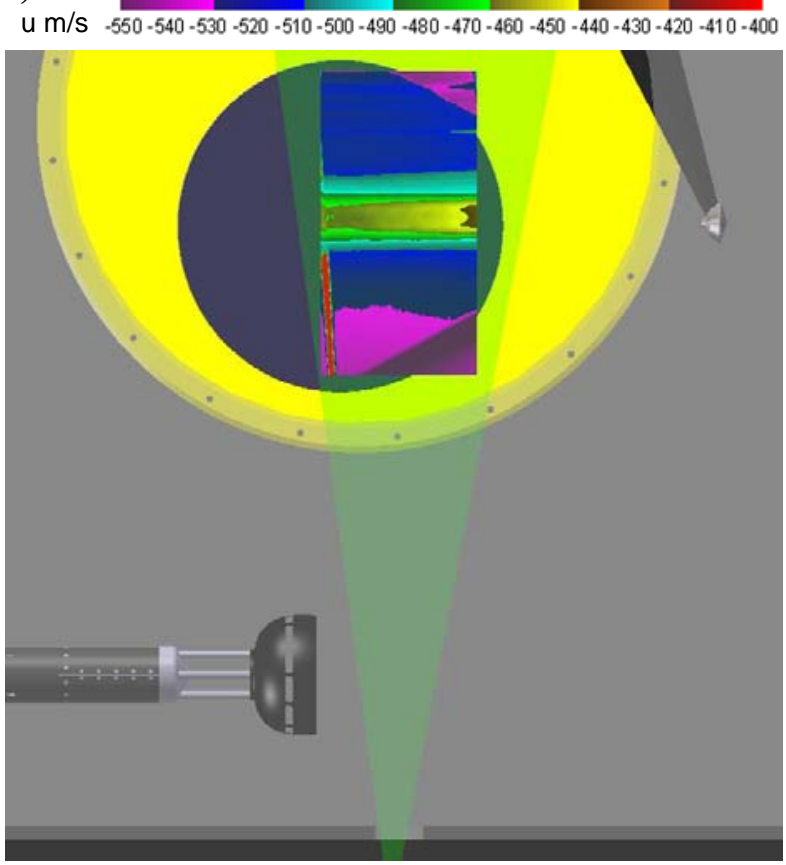

d)
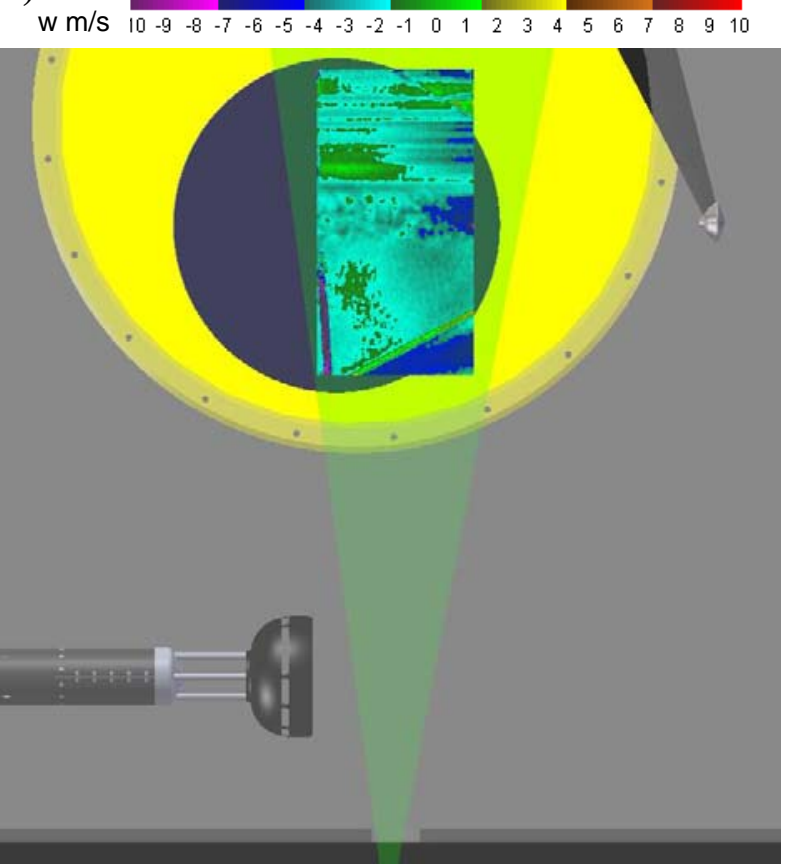

Figure 7: Capsule-only ensemble averaged flow field results at $M$ 2.0, $D_{p}=19 \mathrm{~m}$ : a) Turbulence intensity; b) Axial velocity component; c) Vertical velocity component; d) Cross-stream velocity component. The light sheet passes through the strong bow shock from the canopy near the floor, which refracts the light resulting in a dark line in the PIV light sheet. Artifacts of the un-illuminated region are observed in the lower right corner of the PIV results. 

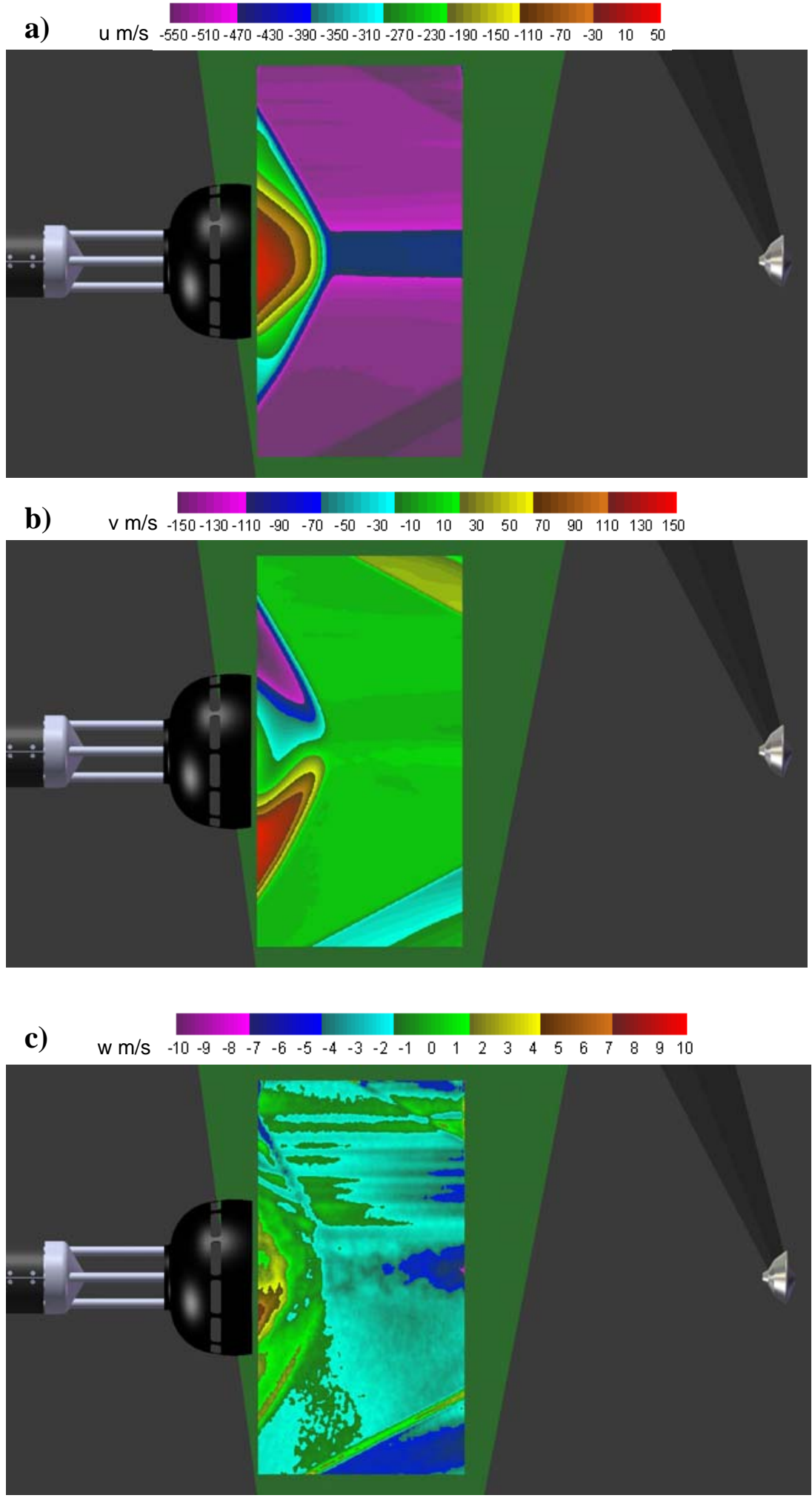

Figure 8: Capsule-Canopy configuration ensemble averaged flow field results: a) Axial velocity component; b) Vertical velocity component; c) Cross-stream velocity component.

American Institute of Aeronautics and Astronautics 
$\rightarrow$ Schlieren Shock Location $\rightarrow$ AF measurement

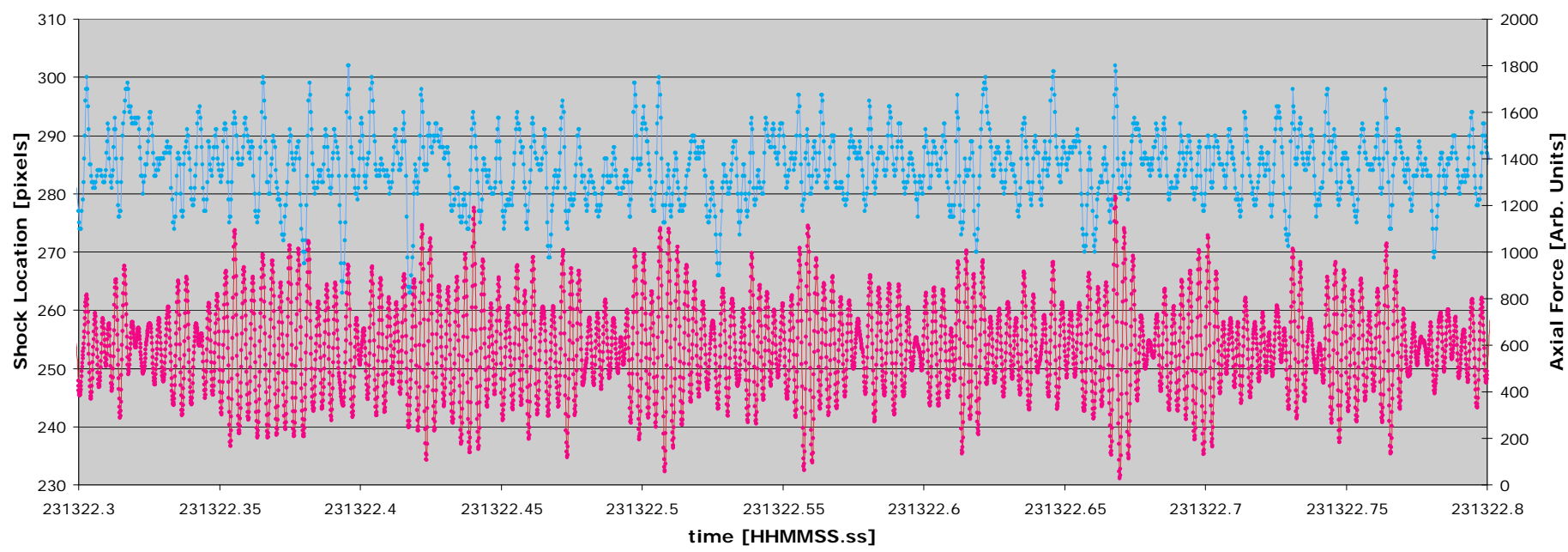

Figure 9: Comparison of shock locations determined from Schlieren images at $4 \mathrm{kHz}$ (top) versus axial force balance readings (bottom), which were recorded at $10 \mathrm{kHz}$. The shock locations are given in units of pixels and the axial force balance readings are in arbitrary units. The force balance readings indicate many more oscillations in the shock motion.

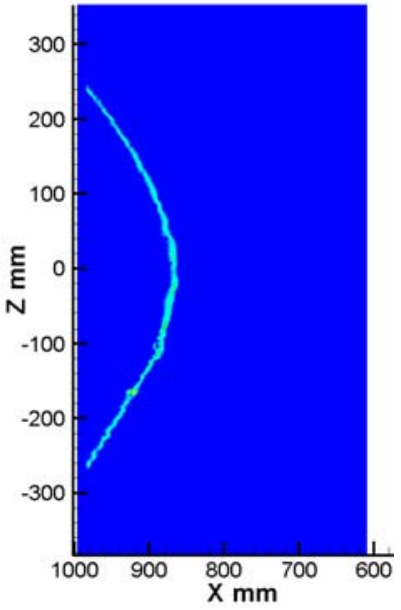

Figure 10: Canopy shock profile determined from velocity vector map.

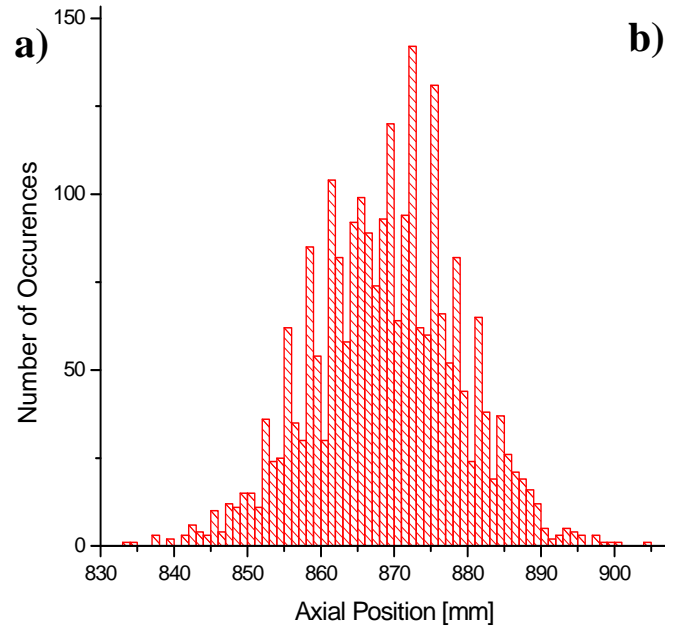

b)

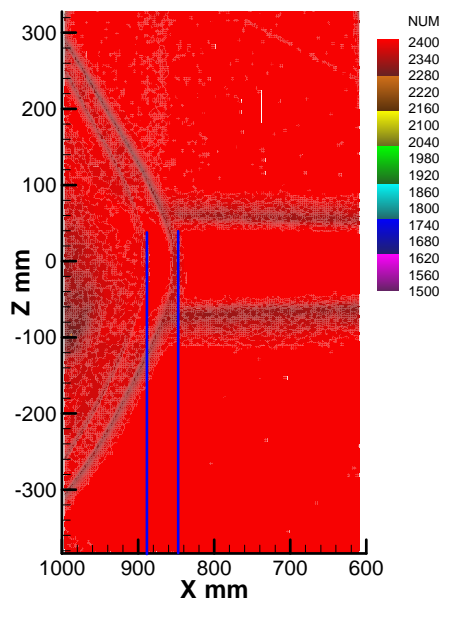

Figure 11: a) PDF of the canopy shock location for a 2400 velocity vector map data set; b) plot of the number of valid measurements at each grid point across the 2400 point record - where the dark regions clearly depict the min and max axial excursions of the shock. 

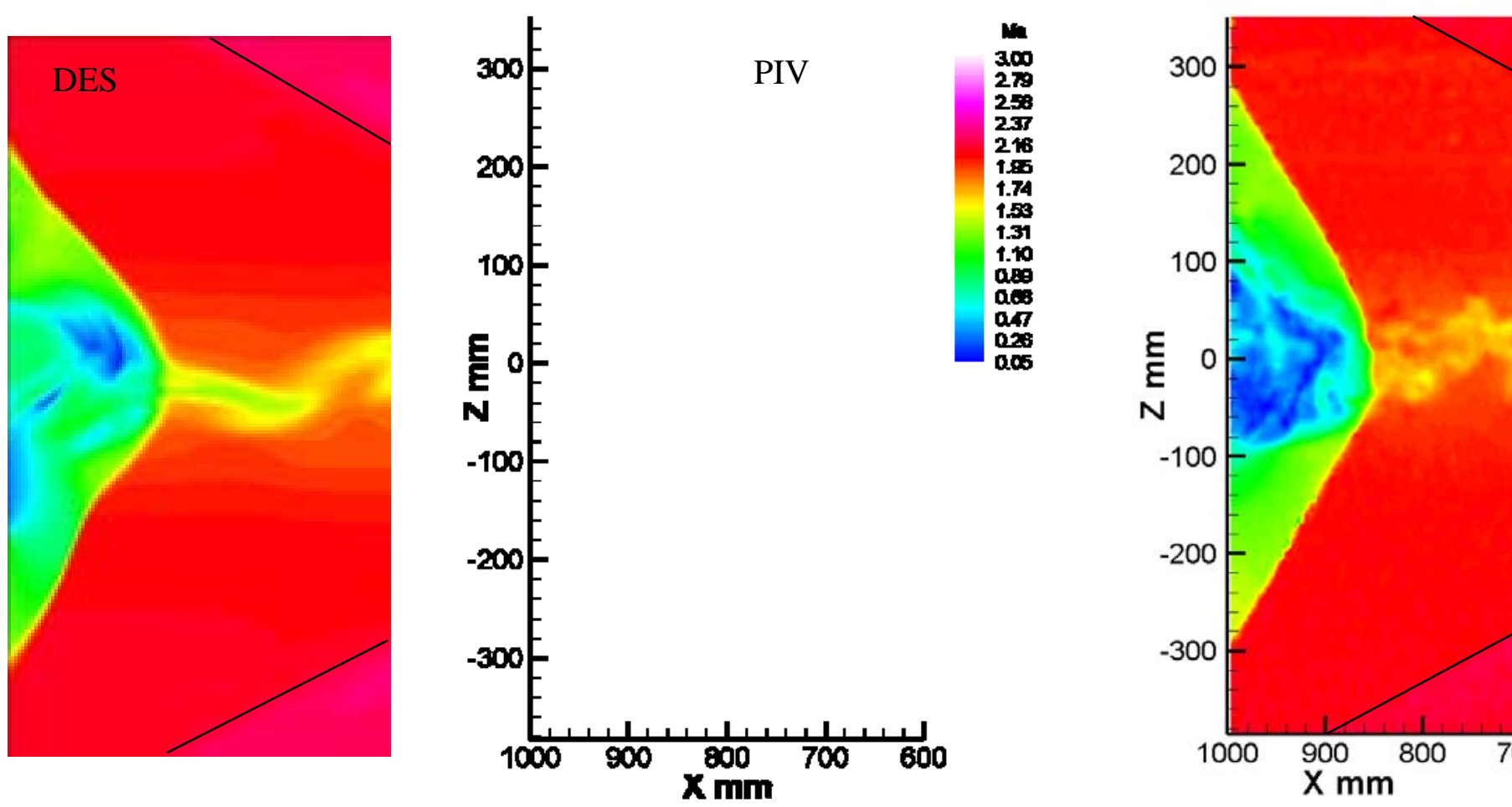

Figure 12: Comparison of DES simulation with the PIV results plotted as Mach number. The black lines denote the capsule wake shock locations. The angle of the canopy wake shocks are symmetric in the DES result and asymmetric in the PIV result, due to the wake of the capsule ceiling strut. 